\title{
Shape-shifting Davydov's ideas for early number learning in South Africa
}

\author{
Hamsa Venkat ${ }^{1,2}$ (D) Mike Askew ${ }^{3} \cdot$ Samantha Morrison $^{4}$
}

Accepted: 25 September 2020 / Published online: 14 November 2020

(C) The Author(s) 2020

\begin{abstract}
In this paper, we share details of a South African early grades' number intervention informed by aspects of Davydov's writing on early number teaching and learning. A key part of Davydov's approach to early number teaching involves starting with attention to relationships between quantities rather than with counting. The Structuring Number Starters (SNS) intervention focused - over a nine-year period - on supporting early grades' students to move beyond the calculating-by-counting approaches that are prevalent in South Africa. In attending to this focus, the intervention shifted increasingly towards an emphasis on relationships between quantities, though not in the same format or task sequence as advocated by Davydov. The contextual and cultural features that led to our adaptations - or shape-shifting - are highlighted in this paper. We interrogate key aspects of Davydov's approaches to early number teaching in relation to key features typical of South African classroom mathematics teaching in order to understand the evolution of the SNS initiative. Quasi-longitudinal interview-based assessment data available from a cross-attainment sample of students in 2011, 2014 and 2018 indicate shifts over time from calculating-by-counting to calculating-by-structuring. These outcomes point to successes with moves into increasingly structured ways of working with early number, but suggest also that these successes may be contingent on some fluency with forward and backward number word sequences. The outcomes suggest that it is feasible to explore interventions directing attention to early number structure from the outset in larger scale studies.
\end{abstract}

Keywords Davydov $\cdot$ Number $\cdot$ Structure $\cdot$ Base-ten $\cdot$ Counting $\cdot$ South Africa

\section{Introduction}

In the South African state education system, the prevalence and persistence of counting-based strategies - counting in ones or counting in multiples to calculate answers to additive and

Hamsa Venkat

hamsa.venkatakrishnan@wits.ac.za

Extended author information available on the last page of the article 
multiplicative number problems - is a widely described phenomenon (Hoadley, 2007; Schollar, 2008). The persistence of counting-based strategies has been described, in Van den Heuvel-Panhuizen's (2008) terms, as showing a lack of moving from calculation-by-counting into calculation-by-structuring.

We have noted in earlier writing that the notion of structure in mathematics fundamentally involves arranging, or re-arranging, the objects being worked with (for example, numbers, variables or expressions) in some mathematically appropriate relation to each other (Venkat, Askew, Watson, \& Mason, 2019). Calculating-by-structuring, therefore, involves leveraging number relationships for more efficient calculation. This can involve, for instance, awareness of the distance relationship between pairs of numbers and using this relationship to choose an efficient way of calculating (e.g., 102-97; 102-3). It can also involve awareness of the baseten structure in the decimal number system. This awareness manifests itself in use of the relationships between numbers in a problem and the friendly numbers in terms of the base-ten system (multiples of powers of ten) for efficient calculating (e.g., using bridging through ten to move forward from counting on in ones in problems like $17+8$ to jumping from 17 to 20 , and then jumping the remaining 5 to get 25 ; or using jump strategies for questions like $38+23$, by starting at 38, jumping forward 20 and then jumping forward another 3). Wright, EllemorCollins and Tabor (2012) use the term base-ten thinking to describe children's competence with reasoning with tens and ones in the context of early number in ways that provide foundations for later work with more formal place value.

In a context where ongoing counting in ones is prevalent, with limited evidence of moves to using number relations and base-ten structure, we have become particularly interested in curricular approaches that can support students working with structured versions of number rather than with counted versions of number. It was this interest that led us to Davydov's work on early number teaching, and from this work, into an adaptation of Davydov's approach in the Structuring Number Starters (SNS) project located in South African government schools serving disadvantaged student populations.

Davydov's approach to early number offers attention to quantities in a relational sense rather than in a counting-based sense. Coles (2017), advocating for the relational sense, details the distinctions between these two approaches, and argues that the relational sense of quantity seen in Davydov's curriculum (and in Gattegno's (1974) work) provides a route into an awareness of number and number relationships as simultaneously material and abstract. However, curricular and pedagogical norms in South Africa present counting as the introductory route into number and into calculation, echoing Coles' broader (2017) sentiment that "the predominant narrative in schooling is a counting world" (p. 206).

\section{Theoretical framing}

In an intervention project interested in larger scale implementation, the predominance of counting necessitated, for us, adaptations to Davydov's approach to make it practicable for use at larger scales in classrooms, as direct application was too far from current practices. Cowen (2009), writing in the field of comparative education, notes the shape-shifting that occurs in the context of transnational policy translations. In Cowen's work, shape-shifting refers to "the re-interpretation of educational ideas which routinely occurs with the transfer in space: "the chameleon process"” $(2009$, p. 323). Such international translations have historically been described in the comparative education and policy fields in dichotomized ways. On 
one side, critiques of policy borrowing raise concerns about monolithic views of what counts as best practice that discount local cultures and priorities. On the other side, there is praise for what is seen as evidence-informed policy development based on international standards (Steiner-Khamsi, 2012). Cowen suggests that attention to shape-shifting involves rigorous analysis of both the internal dimensions of the travelling reform, and the relationships between these internal dimensions and the host context and culture. This approach has been followed in studies such as Poole's (2016) analysis of attempts to incorporate formative assessment practices in Shanghai. At a deeper mathematical level, Mellone, Ramploud and Carotenuto (2020) suggest a cultural deconstruction of the original educational intentions of the incoming idea, in order to "inaugurate other interpretative keys of the didactic practice of their own cultural context" (page number forthcoming).

Thus, two questions guide our focus in this paper. Firstly, what kinds of adaptations to Davydov's early number curriculum were needed to align with classroom cultures and conditions in South African classrooms? Second, what kinds of changes in early number outcomes were associated, over time, with the implementation of a shape-shifted Davydovinformed curriculum focused increasingly on number structure?

The notion of shape-shifting functions as the theoretical basis of this paper. We take up the suggestions of Cowen, and Mellone and her colleagues, in considering the shape-shifting involved in our transposition of Davydov's work. We do this with reference to both key aspects of the original approaches, and to our re-interpretations of these approaches in the light of South African evidence on classroom context, conditions and pedagogies. In doing so, the paper offers insights into the interplay between theory and practice and the importance of cultural considerations within this interplay. We begin by examining two positions on early number progression that figure as background to our analyses of key aspects of Davydov's advocated approach to early number teaching. We go on to outline some specificities of the South African curricular and classroom cultural context. This combination of attention to incoming ideas and host culture set the scene for our thinking about the approaches that could be used to engender attention to structure. Student assessment data over time provided insights into shifts in early number working that fed into subsequent changes in our emphases. The learning outcomes are detailed and used to discuss a trajectory of work that has moved increasingly towards Davydov's emphasis on mathematical structure from the outset in the early grades.

\section{Early number progression: Two positions}

Backgrounding our shape-shifting of Davydov's approaches to early number is a debate in the literature on how structural ways of working with number can be supported. This debate centres on two distinct avenues for supporting structural approaches to calculating. In one direction, a substantial body of writing, with a long history, suggests a trajectory in which counting functions as the fundamental action, with counting actions becoming reified and truncated over time around mathematical structures and properties (base-ten structure and commutativity properties, for example) (e.g., Steffe, Cobb \& von Glasersfeld, 1988). This pathway takes in the features delineated in learning to count effectively_-working with one-toone correspondence and the coordination of the ordinal sequence of number words with objects counted among these (Gelman \& Gallistel, 1978). Concrete counting out of objects in direct models of situations (Carpenter, Fennema, Franke, Levi, \& Empson, 1999) gives way 
over time to indexical modes of counting and then increasingly efficient ways of counting (e.g., counting on from the first number or counting on from the larger number in addition contexts, or counting down from the first number, or counting down to the subtrahend number in subtraction contexts). Within and beyond these more efficient one-by-one calculating-bycounting approaches, further efficiencies ensue as the structure of number relationships comes increasingly into view. Eventually, fluent working with these structures leads to a bank of known, or recalled, facts that can then be used to derive further results. Essentially, this perspective suggests a counting-into-structure trajectory.

In another direction, curricula developed on the basis of Davydov's (1990) work suggest, instead, a straight-for-structure trajectory, where comparison of continuous quantities is the fundamental action, rather than starting with counting of sets of discrete objects. In contrast to the counting-discrete-objects first approach, students' early encounters with mathematics in the curriculum developed by Davydov and his colleagues involve non-numerical comparisons of quantities: lengths of strips or capacities of containers, for example. In carefully devised sequences of comparison activities with physical materials, tasks focus on representation of the relationships between the quantities in multiple ways, rather than on calculation of missing quantities or relations. Discussion focuses on various algebraic ways of representing a situation where strip A or capacity A is longer, or greater, than strip B or capacity B: e.g., as $\mathrm{A}>\mathrm{B}$ and $\mathrm{B}<\mathrm{A}$, or that $\mathrm{B}$ plus some quantity $\mathrm{x}$ added to $B$ could make the length or the capacity equal to A. In emphasizing, from the start, relations between entities and the actions required to turn an inequality into an equality, attention to properties of quantitative attributes of objects is foregrounded.

In Davydov's early additive working, the aim is not only the expression of a particular relationship between quantities, but, more importantly, also the production of a range of generalized logical relationship expressions connected to the situation. In this approach, the calculation of missing quantities in particular arithmetic expressions is deferred to later working. Particular expressions are seen, instead, as instantiations of the generalized expressions of actions on quantities that have been produced in earlier working. Schmittau and Morris (2004) offer useful commentaries on the introductory task sequence in grade 1 outlined above involving strip/capacity comparisons, and what this means for the ways in which traditional number tasks are interpreted when they eventually arise. For example, in students' dealing with $4+\ldots=9$, a fundamental link between quantities and the actions involved in equalizing them is noted:

Although this looks like a typical first grade problem, the meaning that the children give to the equation is affected by the prior work with quantities. The number 4 is interpreted as the numerical value of the smaller quantity, and 9 as the numerical value of the larger quantity. There is an inequality relationship between these quantities. In order to move from an inequality to the desired equality relationship, we have to add the difference to the smaller quantity. Children draw an arc between the beginning of the number line and 4 to show the quantity whose numerical value is 4 ; this is the smaller quantity. They draw another arc between 4 and 9 . This length is the difference - the quantity that must be added to the smaller quantity in order to move to an equality relationship.

[... Children interpret statements of equality in terms of relationships between quantities, and in terms of actions on quantities or arithmetic actions on the numerical values of measured quantities. The meanings of the indicated actions and 
relationships are derived from work with quantities, abstracted, and applied to work

with numbers. (Schmittau \& Morris, 2004, p. 67)

In Davydov's approach, the numbers in number sentences like $4+\ldots=9$ therefore function as references to quantitative attributes of material objects. This contrasts with the typical introductory representations seen in South African early grades' texts in which numbers are linked to counts of discrete objects, and in pedagogy that frequently involves oral recitations of number with no material referents. Thus, current practices in South Africa are more aligned to the counting-into-structure approach than the straight-for-structure position. We return to this point in discussion of the approach followed in the SNS project.

\section{Studying shape-shifting}

Studying the shape-shifting of external initiatives requires two analyses. The first is an analysis of the incoming initiative: Davydov's approach to early number teaching in our case, and the goals, rationales and approaches outlined not only in its original form, but also in subsequent international interpretations. Second, an analysis of the curricular and cultural milieu of the host context is required to make sense of the adaptations and interpretations of the travelling intervention.

\subsection{Davydov's approach to early number teaching}

We focus here on selected aspects of the early number teaching approach outlined above that involve concrete comparison activities linked with a range of algebraic representations of the relationship between the quantities. Specifically, we note two aspects that have been marked in several international initiatives borrowing Davydov's approaches, and that were of particular interest to us, given the South African concerns that are discussed in more detail below. These are focus on structural relations, and the use of non-numerical representations of quantities. We consider these aspects, their underlying assumptions, and implications of these aspects for thinking about additive relations teaching and learning.

Focus on structural relation Davydov's method of comparing concrete quantities and then representing the comparison algebraically in a range of structural representations of the comparative relation differs from the approaches suggested by other research traditions for additive problem-solving. This emphasis on structural relation between quantities rather than enumerating quantities has been taken up in several Davydov-inspired studies internationally (Polotskaia, 2017; Venenciano \& Heck, 2016). The attention to multiple ways of representing single additive relations contrasts with the problem-solving approaches promoted, for example, in Carpenter et al.'s (1999) work on additive situations. In that work, while there is attention to setting up initial models of the relations implied in problem situations, there is less subsequent emphasis on alternative structural representations of those relations. Instead, attention shifts to solving the specific problem at hand by calculating the missing value. This problem-solving approach is associated, in Carpenter et al.'s (ibid) work, with hierarchies of difficulty. Missing addend/subtrahend and missing start problems are noted as harder for children to solve than missing result problems. In 
contrast, Polotskaia (2017) points to a closing of the extent of such differences in difficulty that arises through working on representing the additive structure in different ways.

While this contrast between arithmetical problem-solving and structural re-arranging has been noted in the Davydov-linked literature (e.g., Polotskaia, 2017), the more salient contrast for us, and following Coles (2017), is between pedagogic approaches launching from a base in structure to those launching from a base in counting.

Non-numerical representation A key element of Davydov's early mathematical working is that, initially, comparisons of attributes of objects such as length or capacity occur with no reference to numbers at all. Initial diagrammatic models of similar or different quantities are produced using line segments of similar or different lengths. The relationship between these lengths is then algebraically recorded, as described above, using letters and equality/inequality signs, with discussion emphasizing that the abstracted symbolic record can stand in this way for any comparison relationship. The results of interventions beginning in this way suggest successes with appropriation of reversibility and irreversibility, and transitivity properties. Plus and minus signs are then introduced to represent changes in material quantities, which come to be represented in generalized relational formats. Line segment models continue to be used to facilitate awareness of ways to move from inequality to equality through which students "learn to compare and write out equations ('If $\mathrm{a}<\mathrm{b}$, then $\mathrm{a}+\mathrm{x}=\mathrm{b}$ or $\mathrm{a}=\mathrm{b}-\mathrm{x}$ ') and then to determine $\mathrm{x}$ as the function of other elements in the formula" (Davydov, 1990, p. 168). Attention to commutativity and transitivity properties then ensues. It is only after the student has appropriated this sequence of properties in symbolic, non-numerical form that numbers are introduced in the following way:

In our course the teacher, relying on the knowledge previously acquired by the children, introduces number as a particular case of the representation of a general relationship of quantities, where one of them is taken as a measure and is computing the other. A number is obtained by the general formula $\mathrm{A} / \mathrm{C}=\mathrm{N}$ where $\mathrm{N}$ is any number, $\mathrm{A}$ is any object represented as a quantity, and $\mathrm{C}$ is any measure. (Davydov, 1990, p. 169)

In such tasks and resultant student activity, number emerges as a multiplicative concept, as a ratio of the initial length to the measuring unit length. A consequence of this approach is that the resulting length of the measured quantity is dependent on the length of the measuring unit, bringing the concept of the unit sharply into focus, as well as the inverse relation between the size of the unit and the number that represents a measured length. Another consequence is that rational numbers arise through this activity as easily as the natural numbers, avoiding the significant conceptual reorganization of number properties that has been noted as necessary in more traditional instruction where the introduction of rational numbers is delayed (Schmittau, 2003).

Reflecting on incoming initiative Looking at Davydov's work as outsiders, an important point to note was that Davydov's students appeared to have prior familiarity with the number word sequence, and were able to call this sequence up unproblematically when eventually required to enumerate the number of units in the measured quantity. This point was notable because in our baseline interviews with 7-year-old grade 2 students at the start of the SNS project, this kind of fluency with number word sequences was far from universal (detailed in the following section). While not mentioned explicitly in Davydov's work, the implication of children being able to enumerate the number of unit replications is that knowledge of the 
number word sequence is part of the everyday knowledge repertoire of students acquired prior to the start of formal schooling.

One way of demarcating the international Davydov-related incorporations of early number teaching is to divide those structure-oriented approaches that have taken on the non-numerical introduction to quantitative comparison, from approaches that have adopted the attention to structure but broken with the algebraic representation to use numerical representations of quantity instead. Jean Schmittau's implementation of Davydov's curriculum in the USA (see Morris, 2000; Schmittau \& Morris, 2004) and the work of the Measure Up team in Hawaii (Dougherty \& Slovin, 2004; Venenciano \& Heck, 2016) both fall into the first fidelity of implementation category. In contrast, in Polotskaia's (2017) work in Canada and Mellone and Tortora's (2017) work with older children in Italy, numbers are used in structural comparison activities. Both of these latter teams cite children's prior familiarity with number-based approaches to additive problem-solving in their curriculum and cultural contexts as the reason for working with numerical representations of additive relationships. Given the lack of universal appropriation of verbal number word sequences in our context, we were particularly interested in the rationales offered by others for their decision to work in numerical, rather than algebraic, formats, and also in the approaches they developed with this adaptation in place.

There are other important aspects that are marked in Davydov's work such as working with continuous, rather than discrete, quantities. As our later illustrations of tasks incorporating semi-structured number lines indicate, we did incorporate artifacts presenting number as continuous quantities. However, the continuous/discrete distinction was not the focus of our pedagogic attention in the same way that the calculating-by-counting versus calculating-bystructuring distinction was. Rather, we introduced representations underpinned by continuous length representations of number and focused on the ways in which awareness of number relationships in these representations could be leveraged for attention to structure and more efficient calculation.

In the next section, we overview the orientations towards additive structure in the early grades in the South African curriculum document, and in pedagogy on the ground. We draw on similarities and contrasts with the contexts detailed in other international interpretations of Davydov's work within this overview.

\subsection{South African curriculum and classroom context and cultures}

We have noted already the dominance of concrete counting approaches in South Africa, well into the upper primary grades (grade-appropriate students are 13 years old by grade 7, the last year of primary schooling, but many classes include retainees - students held back due to poor performance). Images from prior research provide graphic evidence of such approaches from across the primary grades - see Fig. 1a-c:

Counting approaches like these were prevalent in the ten partner schools that we began working with in 2011. In administering an individual interview-based early number assessment designed within the Mathematics Recovery programme (Wright, Martland \& Stafford, 2006) to a cross-attainment sample of grade 2 students in 2011, we found that almost three quarters of the overall sample $(74 \%, n=238)$ were working with count-all approaches in the context of early additive situations (in which the whole quantity was less than 20). For example, in calculating $5+4$, a student would count out 5 counters, count out a separate 4 counters, and then count the entire collection from 1 to 9 . 
a

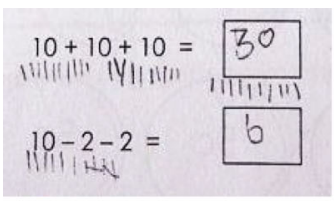

$\mathrm{b}$

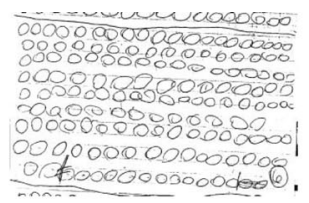

c

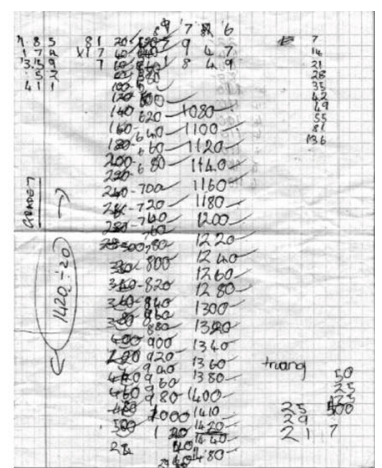

Fig. 1 Counting-based approaches in South Africa. a Weitz and Venkat (2013) Grade 2 student. b Hoadley (2007) Grade 3 student $214+12$ c Schollar (2008) Grade 7 student $1420 \div 20$

In South African writing, this phenomenon has been understood from a range of perspectives. Sociological studies, following Bernsteinian approaches, have interpreted countingbased approaches as concrete in the sense that they are tied to a specific material base. This specific material base has been understood as arising from the ongoing availability and use of unstructured counting resources (bottle tops, cubes or counters) (Ensor et al., 2009). There is also evidence, however, of unit-counting approaches being reinforced by teaching oriented towards solving the problem immediately at hand. Here, both teachers and students treat all examples as highly individual, repeatedly solving every example through count-all procedures, wherein neither procedures nor results are treated as established over time (Venkat \& Naidoo, 2012). Progression from counting into structuring (making use of number relationships and properties for more efficient calculation) is thus limited.

Policy responses to such evidence have taken instructional coverage as a key instrument for ensuring learning progression, and have focused on increased curricular prescription of content, its sequencing and pacing (DBE, 2011). In contrast to this, as we considered intervention project options, we focused on how progression in early number learning was characterized in the curriculum, and how this progression was understood by teachers. In this regard, the overarching curriculum statement about number concept development mentions elements of both counting and structuring (with number relationships involved in building up and breaking down numbers): "learners' number concept is developed through working with physical objects to count collections of objects, partition and combine quantities, skip count in various ways, solve contextual (word) problems, and build up and break down numbers" (DBE, 2011, p. 9).

Subsequent mention of number concept development in the document suggests three avenues for progression: increasing the number range, expanding the types of number being worked with, and moving towards more efficient calculation strategies (DBE, 2011, p. 19). The wording reflects more of the counting-into-structure trajectory, a progression for additive working that can be broadly described in terms of count-all, count-on, count-on-from-larger, calculate efficiently by drawing on number relationships/properties, and using a range of 
known and derived facts (e.g., Wright et al., 2006). Policy recommends attention to mental recall of basic arithmetical facts and consolidation of concepts and, to this end, teachers are expected to conduct daily ten-minute mental starters that open lessons. We noted, however, in our observations of these mental starters in our project schools, frequent collective choral recitation of single unit or multiples-based forward and backward counting sequences across the Foundation Phase grades 1-3 (Askew et al., 2019). Such activities reflected the countingfirst orientation, but provided very little evidence of progression into number structure.

Further to this, and as flagged earlier, in spite of this emphasis on recitation of number sequences, our aforementioned 2011 grade 2 student interviews indicated that children were far from being universally fluent in number word sequences for forward and backward oral counting. Almost a quarter of the students in the 2011 baseline sample $(23 \%, n=238)$ were unable to answer questions asking for the number word after a given number in the 1-10 range without dropping back to count from 1 , and $37 \%$ of this cohort were unable to state the number word before a given number in the same range, again without dropping back to 1 to do so.

Askew et al.'s (2019) empirical analyses of teaching also note limited attention to structure and generality, pointing to difficulties for some teachers in orchestrating the use of manipulatives and inscriptions coherently with their associated talk and/or with the task being worked with. Such phenomena contrast with the skillful and mindful working across concrete comparison activities, and their representation in graphical diagram and algebraic forms in Davydov's descriptions. Indeed, generalized algebraic forms do not feature in the South African curriculum specification across any of the primary grades, and where graphical representations are mentioned (e.g., number lines), they are presented as useful tools for solving specific problems. Askew et al.'s (ibid) analysis notes that connected example sequences are rare in instruction and that the use of general language that might be used to connect examples is also limited. These pedagogical issues are compounded by a provincial primary schooling context in which class sizes are large (50-60 students/class is commonplace), and access to structured resources (resources with the potential to support awareness of part-whole additive relationships and/or base-ten number system relationships) is limited. Additionally, children's reading and language proficiency levels are low (Spaull \& Pretorius, 2019). These features led us to consider adapted options for maintaining the attention to structure that is central to Davydov's approach to address the problems highlighted. We looked for tasks and task sequences that would involve attention to decimal number structure/part-whole structure in the numerical formats that were closer to the existing practice repertoires and curriculum formulation for the teachers we were working with.

In summary, the prevailing counting-based approaches to early number work in South Africa can be understood in terms of two key orientations to mathematics in classroom cultures in the early grades. The first orientation is towards an operational emphasis where counting is used as a means to calculate the answer. The second orientation is towards disconnected ways of working in which examples are dealt with and solved individually and independently. This second orientation effectively sidelines attention to both common structural features of a range of additive situations, and to relational images of additive situations that hold together a suite of numerical or algebraic sentences. While the curriculum specification (DBE, 2011) points to some desired outcomes of attention to structure in terms of more sophisticated calculation strategies and the usefulness of a growing bank of recalled and derived facts (e.g., pp. 104$105)$, it provides limited illustration on how awareness of connections and relations may be fostered in activity sequences. 
In the following section, we analyse the trajectory of the SNS project, and the ways in which the materials developed for use over time (viewed in conjunction with quasilongitudinal data on learning outcomes) have come to be increasingly aligned with Davydov's straight-for-structure approach.

\section{The Structuring Number Starters project}

The SNS project, launched in 2011, was focused on building students' mental mathematical skills underpinned by number sense. One specific aim was to support more children to move beyond the inefficient counting strategies that are so prevalent in South Africa. Between 2011 and 2019, the model of development activity in the SNS project involved a package of tasks for teachers to try with their Foundation Phase classes in the mental starter section, aimed at building students' mental mathematical skills underpinned by number sense.

The SNS intervention worked with grade cohorts of students across the school year, focusing on one grade in each year of the project (see Table 1 for details). The aim was to support teachers to work with short mental mathematics-oriented tasks in the ten-minute mental starter lesson section advocated in the South African curriculum documents to develop mental mathematics. The content for the mental starter is less prescribed than for the main part of any lesson, and so teachers in our project were willing to try things out. Professional development and intervention activity involved a termly workshop with project team members presenting and discussing the suggested tasks with the teachers.

Phase 1 of the project ran from 2011 to 2015 in ten partner government primary schools, and phase 2 from 2016 to 2019 in ten partner government primary schools. All partner schools served disadvantaged student populations. Six of these schools worked with the project across the whole nine-year period. Our work in these six schools with cohorts across the two phases of the project is summarized in Table 1. In 2011, 2014 and 2018, we administered the counting and additive sections of Wright et al.'s (2006) Mathematics Recovery task-based assessment interview instruments to a cross-attainment sample of six students in each of these schools, producing a 36-student sample in each of these years. Given that schooling is frequently the sole site of access to formal learning for disadvantaged students (Young, 2009), examining differences in the profiles of learning outcomes in this quasi-longitudinal sample provided insights into likely differences in the extent of teaching for structure.

In phase 1 (2011-2015), the substantial proportions of students showing limited fluency with the counting number sequence in our 2011 baseline assessments led to a hybrid package of tasks focused on both counting and on number relations. Tasks focused on counting included pair and whole-class games with counting on from numbers other than 1 , and knowing the number words after and before a given number. Our adapted attention to structure

Table 1 Cohorts tracked across years (assessment data collection years highlighted in bold)

Year and grade cohort worked with during intervention

Phase 1 (2011-2015)

Phase 2 (2016-2020)

\begin{tabular}{|c|c|c|c|c|c|c|c|c|}
\hline 2011 & 2012 & 2013 & 2014 & 2015 & 2016 & 2017 & 2018 & 2019 \\
\hline G2 & G3 & G1 & G2 & G3 & No data collected & G1 & G2 & G3 \\
\hline
\end{tabular}


incorporated a combination of tasks and accompanying structured resources such as bead strings, number lines and 100 squares. Examples of tasks and resources used in our work with different grades over the course of the project are detailed in Fig. 2.

Bead string tasks such as shown in Fig. 2 focus on 1-10 quantities in relational ways. For example, seven beads were attended to as five red and two white beads, or as three beads short of ten beads, thus working with numbers in a relational, rather than counted, sense. Teachers were encouraged to support students to build on this and see 1-20 numbers in similarly relational ways on fingers or bead strings rather than as counted quantities, with $5 \mathrm{~s}$ and $10 \mathrm{~s}$ providing relational benchmarks. Teachers and students were encouraged to record the number relationships produced in symbolic number sentence form. In contextual terms, bead strings were a cheaply produce-able resource that could be used to introduce attention to structure and contrast structure-based approaches with counting-based approaches, and lead in to work with number lines.

In line with the curricular increase in number range, the work in grades 2 and 3 focused on making use of base-ten thinking (Wright et al., 2012). In tasks for these grades, attention was on identifying positions of numbers on semi-structured number lines in relation to multiples of ten, rather than, as was the usual practice on number lines, counting all the divisions to arrive at particular numbers. Alongside this, tasks worked on linking number relationships seen in the 1-20 range to higher number ranges, for example, linking knowledge that $6+5=11$ to problems like $36+5=\ldots$. Number lines and part-whole bar models were both used extensively to model the number relationships involved, and oriented towards the appropriation of the structure of the decimal system for supporting efficient calculation.

By 2014, when our second round of student assessment data collection occurred, the proportions of students in the partner schools able to count forwards and backwards and count on/count back with some fluency had increased substantially, with only 1/36 students (under $3 \%$ ) still unable to count forwards fluently or state the number word after a given number in the 1-10 range without dropping back to 1 , and 3/36 (8\%) unable to count backwards/state the number word before numbers in this range (details of assessment, sampling and outcomes follow in the next section). We also had evidence from Morrison's (2018) doctoral study with South African grade 2 students indicating that attention to base-ten thinking across key structured representations of number such as bead strings, number lines and 100 squares was associated with substantial gains in strategic sophistication, with higher gains further associated with representational flexibility across these resources. This led us, in phase 2, to a straight-for-structure approach in which the counting focused tasks were dispensed with, and

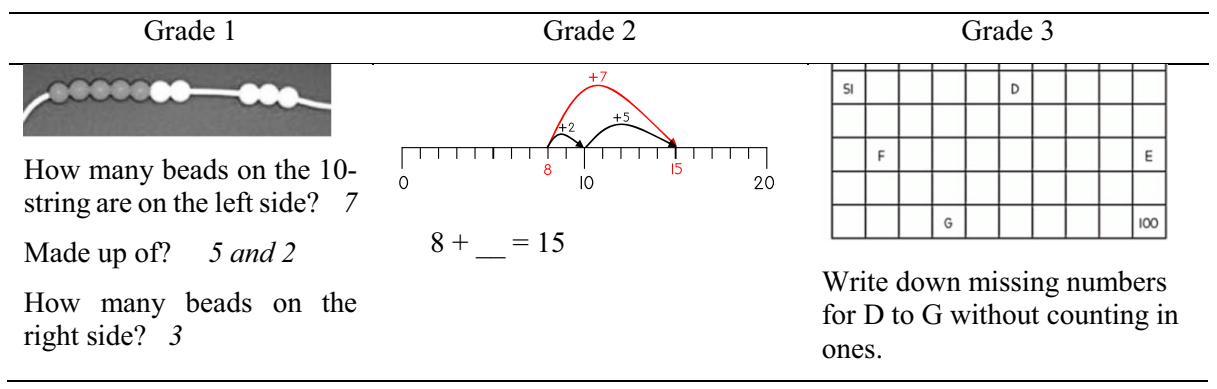

Fig. 2 Number structure-oriented SNS tasks 
the kinds of structure-focused tasks described in Fig. 2 were expanded with increased emphasis on base-ten thinking and connected task sequences focused on using number relationships to derive further results (e.g., $54+28 ; 54+29 ; 55+28$ ).

Our 2018 round of data collection indicated strong signs of moves towards more efficient work with number structure and additive properties - detailed in the following section.

\section{Student assessment tools and findings}

Given the baseline evidence of problems both with fluent counting and with inefficient calculation, we were interested in gathering assessment data over time that could provide insight into both counting and calculation skills. Low levels of reading and writing proficiency rendered written assessments problematic to use. What we found useful were the counting and additive components of the individual interview-based Mathematics Recovery assessments developed by Wright et al. (2006). Items in this assessment allowed us to examine student strategies for the use of Van den Heuvel-Panhuizen's (2008) calculation-by-counting and/or calculation-by-structuring.

Given the problems already noted with counting on/stating the number words after/before numbers in the 1-20 number ranges for substantial proportions of students, we found it useful to split the calculation-by-counting category into two sub-categories: counting all and counting on. Looking at the proportions of early grade 2 students in each of the two counting categories and in the structuring category across the 2011, 2014 and 2018 cross-attainment sample provided a quasi-longitudinal picture of the balance of everyday (counting) and scientific (structuring) approaches in cohorts over time. Given also the evidence of children who were only able to manage counting in concrete settings (i.e., unable to work with additive tasks involving unseen items or symbolic number representations), a lower category based on perceptual counting (where items can be seen or otherwise sensed in some concrete way) was also included. This produced a hierarchy of four categories involving moves from counting to structuring: perceptual counting; calculating-by-counting with count-all; calculating-by-counting with count-on; and calculating-by-structuring.

We administered the counting and additive parts of the Mathematics Recovery assessments to a cross-attainment sample of six students/school cohorts early in grade 2 in each of 2011 (baseline), 2014 and 2018. The 2011 cross-attainment sample was drawn from our more extensive baseline data collection involving six students/class. Grade 2 represents the middle grade of the Foundation Phase in South Africa (grades 1-3, with age-appropriate students between 7 and 9 years old in this phase).

Wright et al.'s (2006) coding of student responses involves looking across sets of items to select the counting or calculating approach that best represents a child's pattern of responses. Their framework for coding rests on a counting-into-structure trajectory with a range of stages based, initially, on increasingly proficient counting, and then into work with structuring through a focus on number relationships and properties. We interpret their stages in relation to the four categories identified above: perceptual counting; calculating-by-counting with count-all; calculating-by-counting with count-on; and calculating-by-structuring. Perceptual counting and the two calculating-by counting categories all fall within the remit of everyday thinking, with number dealt with sequentially through counting rather than structurally through relation. Calculating-by-structuring involves scientific thinking in its use of structure. Recall too that it was in phase 2 (2016-2020) that we moved wholly into the Davydov-informed 
Table 2 Students' test results

\begin{tabular}{lcccl}
\hline Year & Perceptual counting & Counting all & Counting on & Calculating-by-structuring \\
\hline 2011 & $12(33.3 \%)$ & $15(41.7 \%)$ & $8(22.2 \%)$ & $1(2.8 \%)$ \\
2014 & $5(13.9 \%)$ & $9(25 \%)$ & $20(55.6 \%)$ & $2(5.6 \%)$ \\
2018 & $2(5.6 \%)$ & $10(27.8 \%)$ & $15(41.7 \%)$ & $9(25 \%)$ \\
\hline
\end{tabular}

$N=36$ students in each year

straight-for-structure approach, dispensing entirely with tasks and resources focused on supporting counting.

As noted, Wright et al.'s (2006) framework aligns with the counting-into-structure thesis, rather than the straight-for-structure thesis, and thus its use within an intervention that moved increasingly towards the latter thesis may seem contradictory. However, as we have noted, our work in the mental mathematics segment of lessons occurred alongside a curricular and classroom culture context that retained a counting-into-structure orientation. Wright et al.'s assessments provided insights into how the increasing attention to structure in the SNS model in teachers' work was playing through in the six schools that worked with the project across 2011-2019. Calculation-by-structuring, by definition, involves leveraging structure for efficient calculation, and thus, increasing proportions in this category was a specific aim. In Table 2, we summarize the analysis of student responses in relation to the four categories identified above.

Several aspects of these outcomes were of interest in relation to the SNS initiative. Firstly, in 2011, and backing up our evidence of poor counting facility, a third of the 36 students were unable to consistently link a verbal or symbolic number with its associated quantity, either on their fingers or in a diagram (2011: Perceptual counting, 12/36 students). By 2014, this proportion had reduced to 5/36 students, indicating much greater fluency with enumerating in verbal and symbolic number task situations. In 2014, the major change was the substantial increase in the proportions able to use count-on, rather than count-all strategies (2011: Counting on, 8/36 students; 2014: Counting on, 20/ 36 students). In 2018, in the context of our phase 2-modified straight-for-structure approach, there was a much larger incidence of calculating-by-structuring (2018 calculating-by-structuring, 9/36 students). While the small numbers in some cells of Table 2 render statistical tests of significance unsuitable to apply, the close to ten-fold increase in the incidence of calculation-by-structuring points to substantial shifts towards working with number structure, with the near five-fold increase since 2014 suggesting that the major shift into this category occurred in phase 2 of the project as the straight-for-structure model was implemented.

\section{Discussion}

The outcomes of the SNS project over time suggest that the adapted straight-for-structure approach is both viable and promising. While, at one level, the differences in the profile of outcomes can be seen as reflective of the shifts in emphasis towards structuring in the SNS project, in a context of very low levels of moves into using structure, the apparent success of pedagogic attention to structure is an important result.

Beneath this headline, there are further details of interest. In phase 1, the combined attention to counting and to structuring leveraged change from counting all to counting on, with limited moves into structuring. It was in the move to focusing on structure from the start, having seen increasing proportions of students with counting fluency in the interim, that much larger proportions of students 
were seen using structure in their responses. The need for fluency with forward and backward verbal number sequences has not been highlighted in the international literature borrowing Davydov's approaches. These outcomes suggest that it is possible to focus on number structure from the outset in the mental starter section of lessons, with teachers continuing to develop fluency with counting (while also attending to structure) in the context of the stipulated curriculum in the main part of the lesson. Calculating-by-structuring appears to be contingent on some fluency with counting, and this detail is important in the context of policy borrowing, given that international evidence suggests large differences between countries in school entrants' counting skills, quite possibly underpinned by broad differences in what are seen as the everyday competences that children should acquire at home with parental support prior to schooling. Our evidence suggests though that counting fluencies can be developed alongside structural attention within a straight-for-structure approach in mental mathematics working in school.

A final point is that these results were achieved with particular emphasis on number relations within the decimal system from the outset. This shape-shift was motivated by the evidence of children continuing to work with counting approaches that sidelined reference to the affordances of the base-ten system. The responses of the $25 \%$ of learners in the calculating-by-structuring category in 2018 indicated fluent mental working with additive problems in the 1-100 range, accompanied by talk that showed familiarity with and use of base-ten structure.

\section{Concluding comment}

The analysis of shape-shifting in this paper allows for a sharper focus on the particularities of adaptation of a focus on structure in a context where counting approaches predominate. In theoretical terms, we see our work as part of the Davydov lineage in its contrasting of counting-based approaches with structure-based approaches. Our work breaks with Davydov in our focus on numerically based attention to number relationships, rather than starting with non-numerical comparisons of material continuous quantities, and in our emphasis on number relations within the decimal system. These shape-shifts were driven by the need to break with calculation-by-counting in ways that were within reach of the practice repertoires in the system. This system, as detailed in the paper, is firmly rooted in Coles' (2017) reference to a counting world where discrete unit counting is seen as the primary means to solve problems, and the careful coordination of practical resources that might allow for the simultaneous working with number relations as both material and abstract is very rare. In spite of these constraints, the counting/structuring distinction that we encountered in Davydov's work, coupled with his assertion that mathematical work in schools could begin with structuring, has been productive in the trajectory of development of the SNS intervention model. The implementation of the SNS project with its shape-shifted attention to number relations indicates that attention to number structure is within reach of the practice repertoires of the teachers we worked with. Our work is therefore now turning towards straight-for-structure teaching and learning materials and professional development packages for use at provincial levels.

We noted at the start that Mellone, Carotenuto and Ramploud's advice in this volume is that analysis of the cultural transposition of educational initiatives allows host researchers to explore other interpretative keys of the didactic practice of their own cultural context. In our earlier writing, we have analysed gaps in attention to connections and to structure. In our design of the SNS study, informed by Davydov's dismissal of counting in favour of structuring, we have begun the work of re-designing some of the didactic practices of our own cultural context in ways that are attuned to the possibilities and constraints of the ground. 
Acknowledgements The work reported in this paper is located within the South African Numeracy Chair Wits Maths Connect-Primary project at the University of the Witwatersrand.

Funding It is generously supported by the FirstRand Foundation (with the RMB), Anglo American Chairman's fund, the Department of Science and Technology and the National Research Foundation.

Open Access This article is licensed under a Creative Commons Attribution 4.0 International License, which permits use, sharing, adaptation, distribution and reproduction in any medium or format, as long as you give appropriate credit to the original author(s) and the source, provide a link to the Creative Commons licence, and indicate if changes were made. The images or other third party material in this article are included in the article's Creative Commons licence, unless indicated otherwise in a credit line to the material. If material is not included in the article's Creative Commons licence and your intended use is not permitted by statutory regulation or exceeds the permitted use, you will need to obtain permission directly from the copyright holder. To view a copy of this licence, visit http://creativecommons.org/licenses/by/4.0/.

\section{References}

Askew, M., Venkat, H., Abdulhamid, L., Mathews, C., Morrison, S., Ramdhany, V., \& Tshesane, H. (2019). Teaching for structure and generality: Assessing changes in teachers mediating primary mathematics. Vol. 2. Proceedings of the 43rd Conference of the Psychology of Mathematics Education. 7-12 July: Pretoria.

Carpenter, T. P., Fennema, E., Franke, M. L., Levi, L., \& Empson, S. B. (1999). Children's mathematics: Cognitively guided instruction. Portsmouth, NH: Heinemann.

Coles, A. (2017). A relational view of mathematical concepts. In E. de Freitas, N. Sinclair, \& A. Coles (Eds.), What is a mathematical concept? (pp. 205-222). Cambridge, UK: Cambridge University Press. https://oi. org/10.1017/9781316471128.013

Cowen, R. (2009). The transfer, translation and transformation of educational processes: And their shapeshifting? Comparative Education, 45(3), Special Issue 38: Mobility and educational metamorphoses: Patterns, puzzles and possibilities: $315-327$.

Davydov, D. D. (1990). Types of generalization in instruction: Logical and psychological problems in the structuring of school curricula. Soviet Studies in Mathematics Education, Volume 2. Reston, VA: National Council of Teachers of Mathematics.

DBE. (2011). Curriculum and Assessment Policy Statement (CAPS): Foundation phase mathematics, grades R3. Pretoria, South Africa: Department for Basic Education.

Dougherty, B., \& Slovin, H. (2004). Generalized diagrams as a tool for young children's problem solving. In M. J. Hoines \& A. B. Fuglestad (Eds.), Proceedings of the 28th Annual Meeting of the International Group for the Psychology of Mathematics Education, Vol. 2 (pp. 295-302). Bergen, Norway: Bergen University College.

Ensor, P., Hoadley, U., Jacklin, H., Kühne, C., Schmitt, E., Lombard, A., et al. (2009). Specialising pedagogic text and time in foundation phase numeracy classrooms. Journal of Education, 47, 5-30.

Gattegno, C. (1974). The common sense of teaching mathematics. New York, NY: Educational Solutions Worldwide Inc (reprinted 2010).

Gelman, R., \& Gallistel, C. (1978). The child's understanding of number. Cambridge, MA: Harvard University Press.

Hoadley, U. (2007). The reproduction of social class inequalities through mathematics pedagogies in South African primary schools. Journal of Curriculum Studies, 39(6), 679-706.

Mellone, M., Ramploud, A., \& Carotenuto, G. (2020). An experience of cultural transposition of El'koninDavydov curriculum. Educational Studies in Mathematics. https://doi.org/10.1007/s10649-020-09942-7

Mellone, M., \& Tortora, R. (2017). A design study for an Italian fifth grade class following Davydov traces. International Journal for Mathematics Teaching and Learning, 18(2), 240-256.

Morris, A. K. (2000). A teaching experiment: Introducing fourth graders to fractions from the viewpoint of measuring quantities using Davydov's mathematics curriculum. Focus on Learning Problems in Mathematics, 22(2), 33-84.

Morrison, S. (2018). Developing early number learning using math recovery principles. Unpublished doctoral dissertation. University of the Witwatersrand: Johannesburg.

Polotskaia, E. (2017). How the relational paradigm can transform the teaching and learning of mathematics: Experiment in Quebec. International Journal for Mathematics Teaching \& Learning, 18(2), 161-180. 
Poole, A. (2016). 'Complex teaching realities' and 'deep rooted cultural traditions': Barriers to the implementation and internalisation of formative assessment in China. Cogent Education, 3, 1156242.

Schmittau, J. (2003). Cultural-historical theory and mathematics education. In A. Kozulin, B. Gindis, V. S. Ageyev, \& S. S. Miller (Eds.), Vygotsky's educational theory in cultural context (pp. 225-245). Cambridge, UK: Cambridge University Press.

Schmittau, J., \& Morris, A. (2004). The development of algebra in the elementary mathematics curriculum of V. V. Davydov. The Mathematics Educator, 8(1), 60-87.

Schollar, E. (2008). Final report: The primary mathematics research project 2004-2007 - towards evidencebased educational development in South Africa. Johannesburg, South Africa: Eric Schollar \& Associates.

Spaull, N., \& Pretorius, L. (2019). Still falling at the first hurdle: Examining early grade reading in South Africa. In N. Spaull \& J. Jansen (Eds.), South African schooling: The enigma of inequality - a study of the present situation and future possibilities (pp. 147-168). Switzerland: Springer.

Steffe, L. P., Cobb, P., \& von Glasersfeld, E. (1988). Construction of arithmetical meanings and strategies. New York, NY: Springer.

Steiner-Khamsi, G. (2012). Understanding policy-borrowing and lending: Building comparative policy studies. In G. Steiner-Khamsi \& F. Waldow (Eds.), World yearbook of education: Policy borrowing and lending in education (pp. 3-17). London \& New York: Routledge.

Van den Heuvel-Panhuizen, M. (Ed.). (2008). Children learn mathematics: A learning-teaching trajectory with intermediate attainment targets for calculation with whole numbers in primary school. Rotterdam, the Netherlands: Sense Publishers.

Venenciano, L., \& Heck, R. (2016). Proposing and testing a model to explain traits of algebra preparedness. Educational Studies in Mathematics, 92, 21-35.

Venkat, H., Askew, M., Watson, A., \& Mason, J. (2019). Architecture of mathematical structure. For the Learning of Mathematics, 39(1), 13-17.

Venkat, H., \& Naidoo, D. (2012). Analyzing coherence for conceptual learning in a grade 2 numeracy lesson. Education as Change, 16(1), 21-33.

Weitz, M., \& Venkat, H. (2013). Assessing early number learning: How useful is the Annual National Assessment in numeracy? Perspectives in Education, 33(3), 49-65.

Wright, R., Ellemor-Collins, D., \& Tabor, P. (2012). Developing number knowledge: Assessment, teaching and intervention with 7-11 year-olds. London, UK: Sage.

Wright, R. J., Martland, J., \& Stafford, A. K. (2006). Early numeracy: Assessment for teaching and intervention. London, UK: Sage Publications.

Young, M. (2009). What are schools for? In H. Daniels, H. Lauder, \& J. Porter (Eds.), Knowledge, values and educational policy: Vol. 2 Critical perspectives on education (pp. 10-18). London, UK: Routledge.

Publisher's note Springer Nature remains neutral with regard to jurisdictional claims in published maps and institutional affiliations.

\section{Affiliations}

\section{Hamsa Venkat ${ }^{1,2}$ - Mike Askew ${ }^{3}$ - Samantha Morrison ${ }^{4}$}

1 Wits School of Education, University of the Witwatersrand, Room 2, WMC Corridor, Marang Block, St Andrews Road, Parktown, Johannesburg 2050, South Africa

2 School of Education and Communication, Jönköping University, Jönköping, Sweden

3 Wits School of Education, University of the Witwatersrand, Room 3, WMC Corridor, Marang Block, St Andrews Road, Parktown, Johannesburg 2050, South Africa

4 Wits School of Education, University of the Witwatersrand, Room 7, WMC Corridor, Marang Block, St Andrews Road, Parktown, Johannesburg 2050, South Africa 